\title{
Multiple-level power allocation strategy for secondary users in cognitive radio networks
}

\author{
Zhong Chen ${ }^{1,2}$, Feifei Gao ${ }^{2 *}$, Zhengwei Zhang ${ }^{1}$, James C F Li ${ }^{3}$ and Ming Lei ${ }^{3}$
}

\begin{abstract}
In this paper, we propose a new multiple-level power allocation strategy for the secondary user (SU) in cognitive radio (CR) networks. Different from the conventional strategies, where SU either stays silent or transmit with a constant/binary power depending on the busy/idle status of the primary user (PU), the proposed strategy allows SU to choose different power levels according to a carefully designed function of the receiving energy. The way of the power level selection at SU side is optimized to maximize the achievable rate of SU under the constraints of average transmit power at SU and average interference temperature to PU. Simulation results demonstrate that the proposed strategy can significantly improve the throughput of SU compared to the conventional strategies.
\end{abstract}

Keywords: Cognitive radio (CR); Multiple-level power allocation; Spectrum sensing; Statistical reliability; Sensing-based spectrum sharing

\section{Introduction}

Cognitive radio (CR) has recently emerged as a promising technology to improve spectrum utilization and to solve the spectrum scarcity problem [1]. Consequently, spectrum sensing and power allocation play as two key functionalities of a CR system, which involves monitoring the spectrum usage and accessing the primary band under given interference constraints.

The earliest spectrum access approach is the opportunistic spectrum access where secondary user (SU) can only access the primary band when it is detected to be idle [2]. The second approach is the underlay where SU is allowed to transmit beneath the primary user (PU) signal, while sensing is not needed as long as the quality of service (QoS) of PU is protected [3]. The recent approach, sensing-based spectrum sharing, performs spectrum sensing to determine the status of PU and then accesses the primary band with a high transmit power if PU is claimed to be absent, or with a low power otherwise $[4,5]$. These three approaches adopt either constant or binary power allocation at SU which is too 'hard' and limits the performance of SU.

\footnotetext{
*Correspondence: feifeigao@ieee.org

2 Department of Automation, Tsinghua University, State Key Lab of Intelligent Technologies and Systems, Tsinghua National Laboratory for Information Science and Technology (TNList), Beijing 100084, People's Republic of China Full list of author information is available at the end of the article
}

In order to make the power allocation 'softer', we propose a multiple-level power allocation strategy for SU, where the power level used at SU varies based on its receiving energy during the sensing period. It can be easily known that the conventional constant or binary power allocations are special cases of the proposed strategy. The whole strategy is composed of two phases: (i) sensing phase, where the receiving energy is accumulated and the transmit power of SU is decided; (ii) transmission phase, where SU sends its own data with the corresponding power level.

Under the constraints of the average transmit power at $\mathrm{SU}$ and the average interference temperature to $\mathrm{PU}$, the sensing duration, energy threshold, and power levels are optimized to maximize the average achievable rate at SU.

\section{System model}

Consider a CR network with a pair of primary and secondary transceivers as depicted in Figure 1. Let $g_{1}, g_{2}, \gamma$, and $h$ denote the instantaneous channel power gains from the primary transmitter (PT) to the secondary transmitter (ST), from PT to the secondary receiver (SR), from ST to the primary receiver (PR), and from ST to SR, respectively. We consider the simplest case that the channel gains are assumed to be constant and known at the secondary systems and mainly focus on broadcasting the idea of multiple-level power allocation strategy. The idea

\section{是 Springer}

C 2014 Chen et al: licensee Springer. This is an Open Access article distributed under the terms of the Creative Commons

Attribution License (http://creativecommons.org/licenses/by/4.0), which permits unrestricted use, distribution, and reproduction in any medium, provided the original work is properly credited. 


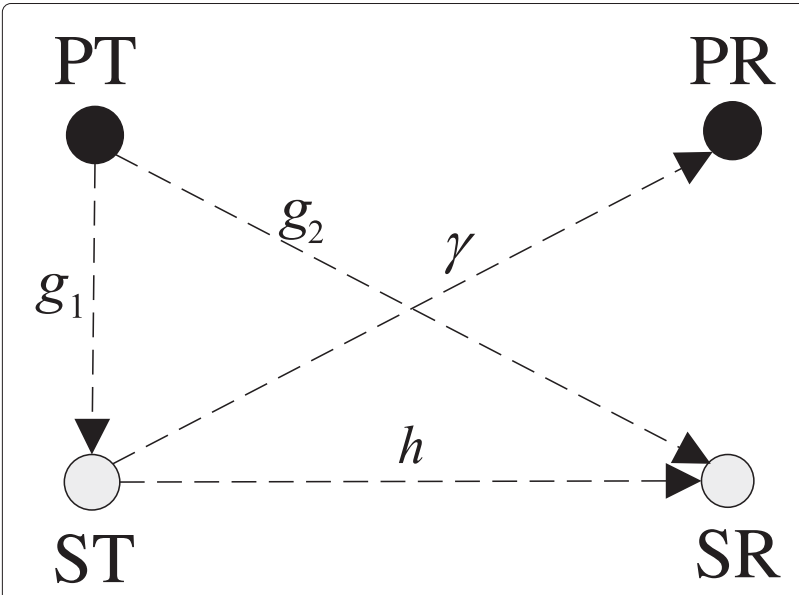

Figure 1 System model of the cognitive radio network.

and the results can be extended to other cases of full/ statistic/partial channel information in the future work.

One data frame of $C R$ is divided into the sensing slot with duration $\tau$ and the transmission slot with duration $T-\tau$. During the sensing slot, ST listens to the primary channel and obtains its accumulated energy. In the conventional schemes, spectrum sensing is performed in this slot and the decisions on the status (active/idle) of the channels are made. When transmitting, ST accesses the primary band with the optimal power in order to maximize the throughput while at the same time keeping the interference to PR.

During the sensing slot, the $j$ th received sample symbol at $\mathrm{ST}$ is

$$
r_{j}= \begin{cases}n_{j}, & H_{0}, \\ \sqrt{g_{1}} e^{j \phi} s_{j}+n_{j}, & H_{1},\end{cases}
$$

where $H_{0}$ and $H_{1}$ denote the hypothesis that PT is absent and present, respectively; $\phi$ is the instant channel phase that is unknown; $n_{j}$ is the additive noise which is assumed to follow a circularly symmetric complex Gaussian distribution with zero mean and variance $N_{0}$, i.e., $n_{j} \backsim$ $\mathcal{N}_{c}\left(0, N_{0}\right) ; s_{j}$ is the $j$ th symbol transmitted from PT. For the purpose of computing the achievable channel rate, the transmitted symbols $s_{j}$ from the Gaussian constellation are typically assumed [4,5], i.e., $s_{j} \backsim \mathcal{N}_{c}\left(0, P_{p}\right)$, where $P_{p}$ is the symbol power. Without loss of generality, we assume that $s_{j}$ and $n_{j}$ are independent of each other.

During the sensing period, the detection statistic $x$ using the accumulated received sample energy can be written as

$$
x=\sum_{j=1}^{\tau f_{s}}\left|r_{j}\right|^{2},
$$

where $f_{s}$ is the sampling frequency at ST. Then the probability density functions (pdf), conditioned on $H_{0}$ and $H_{1}$, are given by [6]

$$
\begin{aligned}
& f\left(x \mid H_{0}\right)=\frac{x^{\tau f_{s}-1} e^{-\frac{x}{N_{0}}}}{\Gamma\left(\tau f_{s}\right) N_{0}^{\tau f_{s}}}, \\
& f\left(x \mid H_{1}\right)=\frac{x^{\tau f_{s}-1} e^{-\frac{x}{N_{0}+g_{1} P_{p}}}}{\Gamma\left(\tau f_{s}\right)\left(N_{0}+g_{1} P_{p}\right)^{\tau f_{s}}},
\end{aligned}
$$

where $\Gamma($.) is the gamma function defined as $\Gamma(x)=$ $\int_{0}^{+\infty} t^{x-1} e^{-t} d t$. Indeed, $f\left(x \mid H_{0}\right)$ and $f\left(x \mid H_{1}\right)$ are both variants of the Gamma distribution.

In the conventional CR, ST compares $x$ with a threshold $\rho$ and makes decision according to $x \underset{H_{0}}{\stackrel{H_{1}}{\gtrless}} \rho$. Specifically,

- In opportunistic spectrum access approach, ST can only access the primary band when $x<\rho$ (it means $\left.H_{0}\right)[7,8]$.

- In sensing-based spectrum sharing, if $x<\rho$, ST transmits with one higher power and otherwise with a lower power (binary power) [9].

- In underly approach, ST transmits with a constant power for all $x$ according to the interference constraint at PU (constant power). No sensing time slot is needed.

\section{Proposed multiple-level power allocation strategy}

It can be easily realized that the conventional constant or binary power of SU does not fully exploit the capability of the co-existing transmission. Motivated by this, we propose a multiple-level power allocation strategy for $\mathrm{SU}$ to improve the average achievable rate.

\section{Strategy of multiple-level power allocation}

Define $\left\{\Re_{1}, \ldots, \Re_{M}\right\}$ as $M$ disjoint spaces of the receiving energy $x$, and $\left\{P_{1}, \ldots, P_{M}\right\}$ as the corresponding allocated power of SU. Then the proposed power allocation strategy can be written as

$$
P(x)=\sum_{i=1}^{M} P_{i} I_{x \in \Re_{i}},
$$

where $I_{A}$ is the indicating function that $I_{A}=1$ if $A$ is true and $I_{A}=0$ otherwise. Note that the conventional power allocation rules are special cases when $M=1$ or 2 .

Using (4), the instantaneous rates of SU with receiving $x$, at the absence and the presence of $\mathrm{PU}$, are given by

$$
\begin{aligned}
& \left.R(x)\right|_{H_{0}}=\sum_{i=1}^{M} \log _{2}\left(1+\frac{P_{i} h}{N_{0}}\right) I_{x \in \Re_{i}}, \\
& \left.R(x)\right|_{H_{1}}=\sum_{i=1}^{M} \log _{2}\left(1+\frac{P_{i} h}{N_{0}+g_{2} P_{p}}\right) I_{x \in \Re_{i}},
\end{aligned}
$$


respectively. Then the average throughput of SU for the proposed multiple-level power allocation strategy using the total probability formula can be formulated as

$$
\begin{aligned}
R=\frac{T-\tau}{T} \sum_{i=1}^{M} & {\left[q_{0} \log _{2}\left(1+\frac{P_{i} h}{N_{0}}\right) p_{i, 0}\right.} \\
& \left.+q_{1} \log _{2}\left(1+\frac{P_{i} h}{N_{0}+g_{2} P_{p}}\right) p_{i, 1}\right],
\end{aligned}
$$

where $q_{0}$ and $q_{1}=1-q_{0}$ are the idle and busy probabilities of the PU respectively; $p_{i, 0}$ and $p_{i, 1}$ are functions of $\tau$ and can be computed from

$$
p_{i, j}=\operatorname{Pr}\left(x \in \Re_{i} \mid H_{j}\right)=\int_{0}^{\infty} I_{x \in \Re_{i}} f\left(x \mid H_{j}\right) d x, j=0,1 .
$$

In order to keep the long-term power budget of SU, the average transmit power, denoted by $\bar{P}$, is constrained as

$$
\frac{T-\tau}{T} \sum_{i=1}^{M} P_{i}\left[q_{0} p_{i, 0}+q_{1} p_{i, 1}\right] \leq \bar{P} .
$$

Moreover, to protect the QoS of PU, an interference temperature constraint should be applied as well. Under (4), the interference is caused only when PU is present. Denoting $\bar{I}$ as the maximum average allowable interference at $\mathrm{PU}$, the average interference power constraint can be formulated as

$$
\frac{T-\tau}{T} \sum_{i=1}^{M} \gamma q_{1} P_{i} p_{i, 1} \leq \bar{I} .
$$

Our target is to find the optimal space division $\left\{\Re_{i}\right\}$, a the power allocation $\left\{P_{i}\right\}$, as well as the sensing time $\tau$ in order to maximize the average achievable rate of $S U$ under the power constraints. The optimization is then formulated as

$$
\begin{aligned}
& \max _{\tau, P_{i}, \Re_{i}} R \\
& \text { s.t. (9), (10), } 0 \leq \tau \leq T, P_{i} \geq 0, \forall i .
\end{aligned}
$$

The term $\frac{T-\tau}{T}$ means that the power constraints occur in the transmission slot. Note that (11) is nonlinear and non-convex over $\tau$. Hence, following $[4,10]$, we will simply use the one-dimensional search within the interval $[0, T]$ to find the optimal $\tau$, whose complexity is generally acceptable as known from $[11,12]$.

\section{The algorithm}

The Lloyd's algorithm is employed here to solve problem (11), where the local convergence has been proved for some cases in one-dimensional space. But in general, there is no guarantee that Lloyd's algorithm will converge to the global optimal [13]. Starting from a feasible solution as the initial value, e.g., subspaces $\left\{\Re_{i}\right\}$ satisfying $p_{i, 0}=\frac{1}{M}$, we repeat the following two steps until the convergence: step 1 - determine the power allocations $\left\{P_{i}\right\}$ given the subspaces $\left\{\Re_{i}\right\}$; step 2 - determine the subspaces $\left\{\Re_{i}\right\}$ given power allocations $\left\{P_{i}\right\}$.

\section{Subspaces design}

First, we demonstrate that the design of the optimal subspace division $\left\{\Re_{i}\right\}$ and power allocation $\left\{P_{i}\right\}$ is equivalent to a modified distortion measure design [14]. Incorporating the power constraints by the Lagrange multipliers $\lambda$ and $\mu$, we define the following distortion measure for optimizing the rate

$$
\begin{aligned}
R\left(x, P_{i}\right)= & q_{0} \log _{2}\left(1+\frac{P_{i} h}{N_{0}}\right) f\left(x \mid H_{0}\right)-\mu q_{1} \gamma P_{i} f\left(x \mid H_{1}\right) \\
& +q_{1} \log _{2}\left(1+\frac{P_{i} h}{N_{0}+g_{2} P_{p}}\right) f\left(x \mid H_{1}\right) \\
& -\lambda P_{i}\left[q_{0} f\left(x \mid H_{0}\right)+q_{1} f\left(x \mid H_{1}\right)\right] .
\end{aligned}
$$

The optimization problem in (11) is equivalent to selecting $\left\{\Re_{i}\right\}$ and $\left\{P_{i}\right\}$ to maximize the average distortion given by

$$
R=\frac{T-\tau}{T} \sum_{i=1}^{M} \int_{x \in \Re_{i}} R\left(x, P_{i}\right) d x .
$$

The optimal subspaces $\left\{\Re_{i}\right\}$ are then determined by the farthest neighbor rule [14] as

$$
\Re_{i}=\left\{x: R\left(x, P_{i}\right) \geq R\left(x, P_{k}\right), \forall k \neq i\right\} .
$$

The following lemma is instrumental to deriving the optimal subspaces $\left\{\Re_{i}\right\}$.

Lemma 1. For $x_{1}<x_{2}<x_{3}$, if $x_{1} \in \Re_{i}, x_{2} \in \Re_{k}$ and $i \neq k$, then $x_{3} \notin \Re_{i}$ must hold.

Proof. Define a function of $x$ as

$$
\begin{aligned}
S_{i, k}(x) & =R\left(x, P_{i}\right)-R\left(x, P_{k}\right) \\
& =\frac{x^{\tau f_{s}-1} e^{-\frac{x}{N_{0}}}}{\Gamma\left(\tau f_{s}\right)}\left[\frac{a_{i, k}}{\left(N_{0}+g_{2} P_{p}\right) \tau f_{s}} e^{\frac{x g_{2} P_{p}}{\bar{N}_{0}\left(N_{0}+g_{2} P_{p}\right)}}+\frac{b_{i, k}}{N_{0}^{\tau f_{s}}}\right],
\end{aligned}
$$

where

$$
\begin{aligned}
a_{i, k}= & q_{1}\left[\log _{2}\left(1+\frac{P_{i} h}{N_{0}+g_{2} P_{p}}\right)-\log _{2}\left(1+\frac{P_{k} h}{N_{0}+g_{2} P_{p}}\right)\right] \\
& -\lambda q_{1}\left(P_{i}-P_{k}\right)-\mu q_{1} \gamma\left(P_{i}-P_{k}\right), \\
b_{i, k}= & q_{0}\left[\log _{2}\left(1+\frac{P_{i} h}{N_{0}+g_{2} P_{p}}\right)-\log _{2}\left(1+\frac{P_{k} h}{N_{0}+g_{2} P_{p}}\right)\right] \\
& -\lambda q_{0}\left(P_{i}-P_{k}\right) .
\end{aligned}
$$

From $x_{1} \in \Re_{i}, x_{2} \in \Re_{k}$ and (14), we know that $S_{i, k}\left(x_{1}\right)>0$ and $S_{i, k}\left(x_{2}\right)<0$. In (14), the sign of $S_{i, k}(x)$ is decided by $\frac{a_{i, k}}{\left(N_{0}+g_{2} P_{p}\right)^{\tau s}} e^{\frac{x g_{2} P_{p}}{N_{0}\left(N_{0}+g_{2} P_{p}\right)}}+\frac{b_{i, k}}{N_{0}^{\tau_{s}}}$ which is a strictly monotonic 
function. Thus, for any $x_{3}>x_{2}$, there are $S_{i, k}\left(x_{3}\right)<0$ and $x_{3} \notin \mathfrak{R}_{i}$.

Proposition 1. $\Re_{i}, i=1, \ldots, M$ are continuous intervals and satisfy $\bigcup_{i=1, \ldots, M} \Re_{i}=[0, \infty]$.

Proof. The proof can be easily obtained from the law of contradiction. Assuming that $\Re_{i}$ has more than two noncontinuous intervals, it is contradicted with Lemma 1. This proposition is instrumental to obtaining the explicit formulation of $\Re_{i}$.

Define $M+1$ thresholds $\eta_{0}, \eta_{1}, \ldots, \eta_{M}$ with $\eta_{0}=0$, $\eta_{M}=+\infty$. Thus, $\Re_{i}$ corresponds to one of $\left[\eta_{j-1}, \eta_{j}\right), j=$ $[1, \ldots, M]$. Based on Lemma 1 , we can calculate $\eta_{j}$ sequentially and assign $\left\{\Re_{i}\right\}$ in Algorithm 1 . The answer of $x_{k}$ that satisfies $S_{i, k}\left(x_{k}\right)=0$ is given by

$$
x_{k}=\frac{N_{0}\left(N_{0}+g_{2} P_{p}\right)}{g_{2} P_{p}} \cdot \ln \left(\frac{-b_{i, k}\left(N_{0}+g_{2} P_{p}\right)^{\tau f_{s}}}{a_{i, k} N_{0}^{\tau f_{s}}}\right) \text {. }
$$

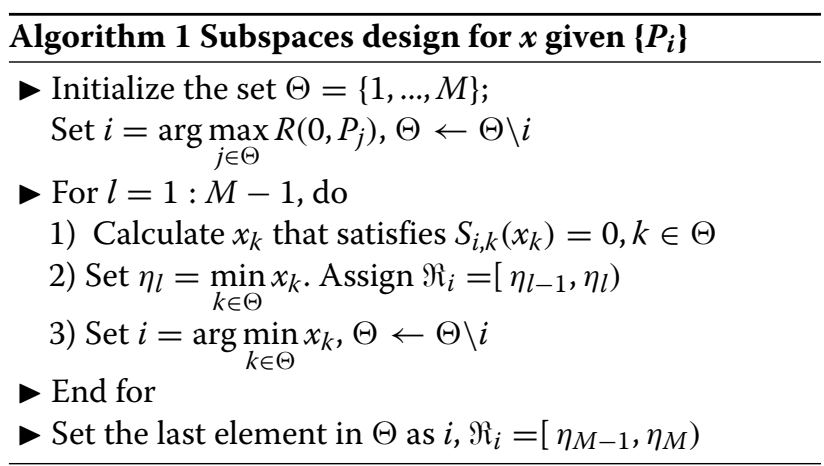

\section{Power allocation}

After obtaining the threshold $\eta_{i}$, the probabilities $p_{i, j}$ in (11) can be explicitly expressed as

$$
p_{i, j}=\int_{\eta_{i-1}}^{\eta_{i}} f\left(x \mid H_{j}\right) d x, i \in[1, \ldots, M], j=0,1 .
$$

Let us first write the lagrangian $L\left(P_{i}, \lambda, \mu\right)$ for problem (11) under the constraints (9) and (10) as

$$
\begin{aligned}
L\left(P_{i}, \lambda, \mu\right)= & R+\lambda\left(\bar{P}-\frac{T-\tau}{T} \sum_{i=1}^{M} P_{i}\left[q_{0} p_{i, 0}+q_{1} p_{i, 1}\right]\right) \\
& +\mu\left(\bar{I}-\frac{T-\tau}{T} \sum_{i=1}^{M} q_{1} \gamma P_{i} p_{i, 1}\right)
\end{aligned}
$$

where $\lambda, \mu \geq 0$ are dual variables corresponding to (9) and (10). The Lagrange dual optimization can be formulated as

$$
\min _{\lambda \geq 0, \mu \geq 0} g(\lambda, \mu) \triangleq \sup _{P_{i} \geq 0} L\left(P_{i}, \lambda, \mu\right) .
$$

$$
\text { In (11), } \frac{\partial^{2} R}{\partial^{2} P_{i}}=-\frac{T-\tau}{T}\left\{\frac{\log _{2}(e) q_{0} p_{i, 0}}{\left(P_{i}+N_{0} / h\right)^{2}}+\frac{\log _{2}(e) q_{1} p_{i, 1}}{\left(P_{0}+\left(N_{0}+g_{2} P_{p}\right) / h\right)^{2}}\right\}<
$$
0 , and $\frac{\partial^{2} R}{\partial P_{i} \partial P_{j}}=0, i \neq j$. Since the constraints are linear functions, problem (11) is concave over $P_{i}$. Thus the optimal value $P_{i}$ of problem (18) is equal to that of (11), and we can solve (18) instead of (11). From (18), we have to obtain the supremum of $L\left(P_{i}, \lambda, \mu\right)$. Taking the derivative of $L\left(P_{i}, \lambda, \mu\right)$ with respect to $P_{i}$ leads to

$$
\begin{aligned}
\frac{\partial L\left(P_{i}, \lambda, \mu\right)}{\partial P_{i}}=\frac{T-\tau}{T}\{ & \frac{\log _{2}(e) q_{0} p_{i, 0}}{P_{i}+N_{0} / h}+\frac{\log _{2}(e) q_{1} p_{i, 1}}{P_{i}+\left(N_{0}+g_{2} P_{p}\right) / h} \\
& \left.-\lambda\left[q_{0} p_{i, 0}+q_{1} p_{i, 1}\right]-\mu q_{1} \gamma p_{i, 1}\right\} .
\end{aligned}
$$

By setting the above equation to 0 and applying the constraint $P_{i} \geq 0$, the optimal power allocation $P_{i}$ for given Lagrange multipliers $\lambda$ and $\mu$ is computed as

$$
P_{i}=\left[\frac{A_{i}+\sqrt{\triangle_{i}}}{2}\right]^{+},
$$

where $[x]^{+}$denotes max $(0, x)$, and

$$
\begin{gathered}
A_{i}=\frac{\log _{2}(e)\left[q_{0} p_{i, 0}+q_{1} p_{i, 1}\right]}{\lambda\left[q_{0} p_{i, 0}+q_{1} p_{i, 1}\right]+\mu q_{1} \gamma p_{i, 1}}-\frac{2 N_{0}+g_{2} P_{p}}{h}, \\
\triangle_{i}=A_{i}^{2}+\frac{4}{h}\left\{\begin{array}{c}
\frac{\log _{2}(e)\left[q_{0} p_{i, 0}\left(N_{0}+g_{2} P_{p}\right)+q_{1} p_{i, 1} N_{0}\right]}{\lambda\left[q_{0} p_{i, 0}+q_{1} p_{i, 1}\right]+\mu q_{1} \gamma p_{i, 1}} \\
\left.-\frac{N_{0}\left(N_{0}+g_{2} P_{p}\right)}{h}\right\} .
\end{array}\right.
\end{gathered}
$$

Proposition 2. The power allocation functions $P_{i}$ are non-increasing over $i$.

Proof. First, from (3), we have

$$
\frac{f\left(x \mid H_{1}\right)}{f\left(x \mid H_{0}\right)}=e^{\frac{x g_{1} P_{p}}{N_{0}\left(N_{0}+g_{1} P_{p}\right)}}\left(\frac{N_{0}}{N_{0}+g_{1} P_{p}}\right)^{\tau f_{s}},
$$

and obviously it is an increasing function over $x$. Through some simple manipulations, the monotonicity of $A_{i}$ is equivalent to the monotonicity of the following term:

$$
C_{i}=\frac{1+\frac{q_{1}}{q_{0}} \frac{p(i, 1)}{p(i, 0)}}{1+(1+\mu \gamma / \lambda) \frac{q_{1}}{q_{0}} \frac{p(i, 1)}{p(i, 0)}} .
$$


From (23), we can get that

$$
\frac{p(i, 1)}{p(i, 0)}>\frac{p(i+1,1)}{p(i+1,0)}, \forall i
$$

Jointly from (24) and (25), we know that $A_{i}$ is a decreasing function over $i$. The monotonicity of $\frac{4}{h}\left\{\frac{\log _{2}(e)\left[q_{0} p_{i, 0}\left(N_{0}+g_{2} P_{p}\right)+q_{1} p_{i, 1} N_{0}\right]}{\lambda\left[q_{0} p_{i, 0}+q_{1} p_{i, 1}\right]+\mu q_{1} \gamma p_{i, 1}}-\frac{N_{0}\left(N_{0}+g_{2} P_{p}\right)}{h}\right\}$ is equivalent to the monotonicity of the following term:

$$
D_{i}=\frac{1+\frac{N_{0}}{N_{0}+g_{2} P_{p}} \frac{q_{1}}{q_{0}} \frac{p(i, 1)}{p(i, 0)}}{1+(1+\mu \gamma / \lambda) \frac{q_{1}}{q_{0}} \frac{p(i, 1)}{p(i, 0)}} .
$$

Similarly, we get that $\triangle_{i}$ is a decreasing function over $i$. Thus, from (20), we can conclude that $P_{i}$ is a nonincreasing function with respect to $i$.

Remark 1. Proposition 2 shows that at smaller $x$, the probability of PU being busy is smaller, so SU can use higher transmit power to better exploit the primary band. On the other hand, at the larger $x$, lower transmit power should be used to prevent harmful interference to PU. Thus, the proposed multiple-level power allocation strategy can also be defined on the probability of PU being busy.

Subgradient-based methods are used here to find the optimal Lagrange multipliers $\lambda$ and $\mu$, e.g., the ellipsoid method and the Newton's method [15]. The subgradient of $g(\lambda, \mu)$ is $[C, D]^{T}$, where

$$
\begin{aligned}
& C=\bar{P}-\frac{T-\tau}{T} \sum_{i=1}^{M} \bar{P}_{i}\left[q_{0} p_{i, 0}+q_{1} p_{i, 1}\right] \\
& D=\bar{I}-\frac{T-\tau}{T} \sum_{i=1}^{M} q_{1} \gamma \bar{P}_{i} p_{i, 1}
\end{aligned}
$$

while $\bar{P}_{i}$ is the optimal power allocation for fixed $\lambda$ and $\mu$ [16]. Finally, we summarize the algorithm that computes the sensing time and multiple-level power allocations in Algorithm 2.

\section{$\overline{\text { Algorithm } 2 \text { Sensing time and multiple-level power }}$} allocations

For each $\tau$ in $[0, T]$, do

1) Initialize $\lambda, \mu, \eta_{i}$ satisfying $p_{i, 0}=\frac{1}{M}$

2) Repeat until $\left\{\Re_{i}\right\}$ converge:

- Get $\left\{P_{i}\right\}$ using (20); Update $\lambda$ and $\mu$ using the subgradient-based method; Until $\lambda$ and $\mu$ converge

- Update $\left\{\Re_{i}\right\}$ using Algorithm 1

End for

- Optimal parameters: $\tau^{*}=\arg \max R\left(\tau, P_{i}, \Re_{i}\right)$,

$\left(P_{i}^{*}, \Re_{i}^{*}\right)=\left.\left(P_{i}, \Re_{i}\right)\right|_{\tau=\tau^{*}}$

Remark 2. All computations are performed offline and the resulting power control rule is stored in a look-up table for real-time implementation. Thus, the computational complexity is not significant.

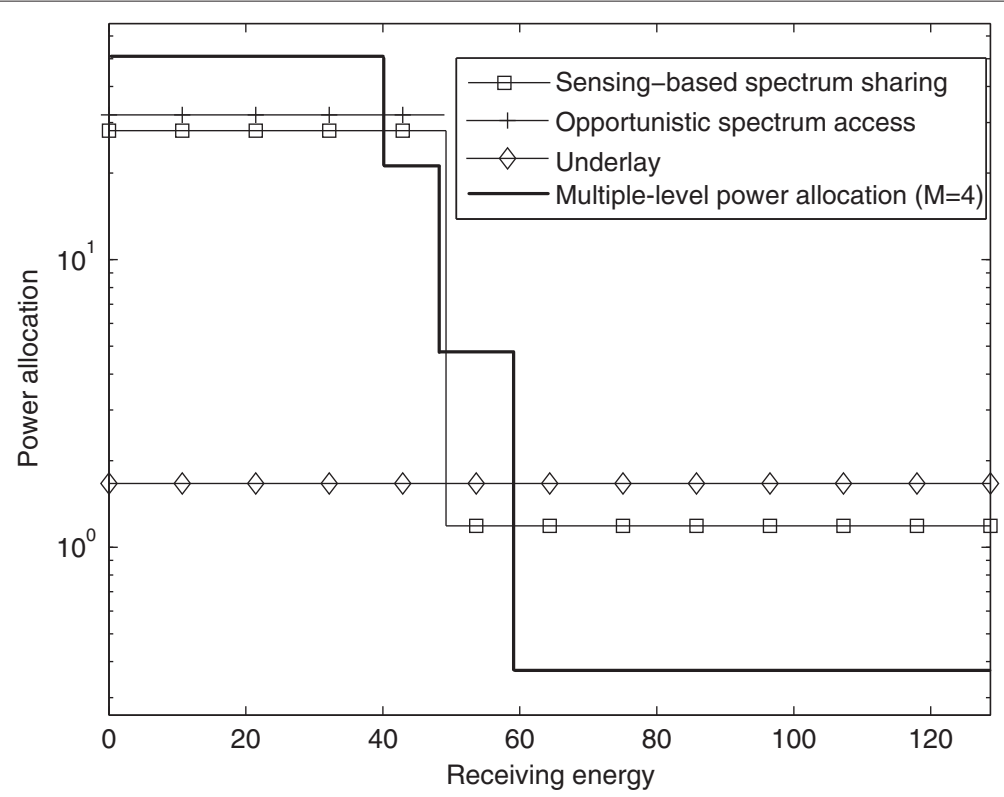

Figure 2 Power allocations under the conventional and proposed strategies. 


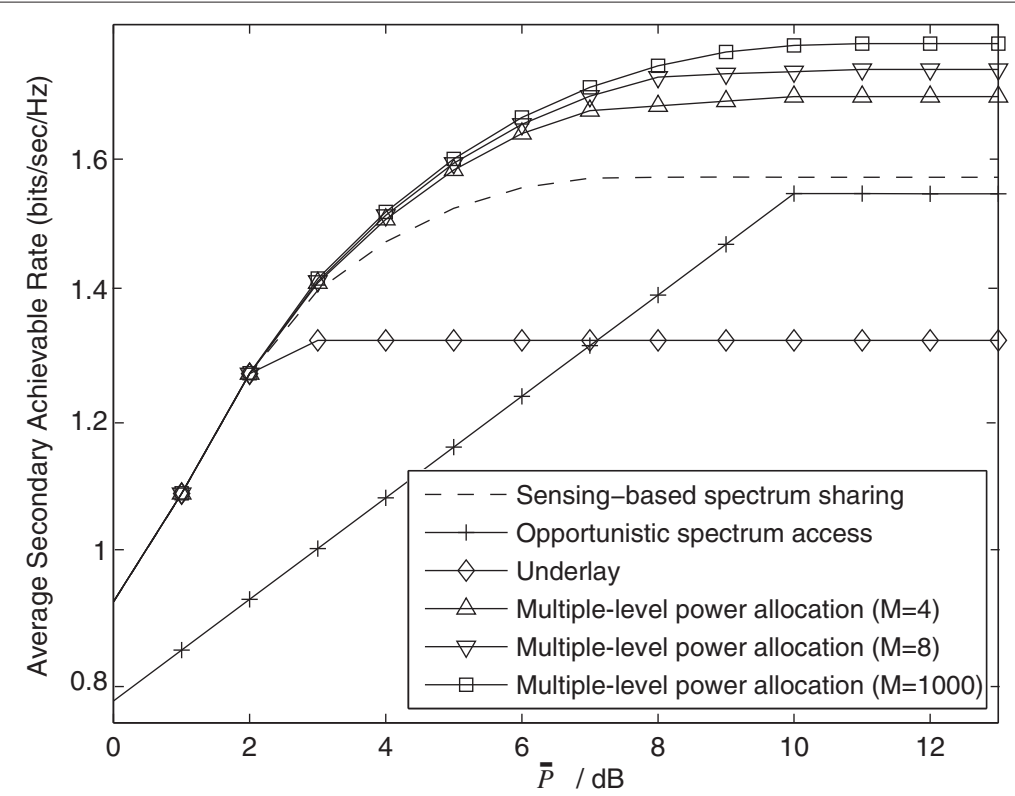

Figure 3 Secondary achievable rate vs. $\bar{P}$.

\section{Simulation results}

In this section, simulations are performed to evaluate the proposed multiple-level power allocation strategy in a CR system where the system parameters similar to the references $[4,5,10]$ are used. The frame duration is taken as $T=100 \mathrm{~ms}$ and the sampling frequency $f_{s}=1 \mathrm{MHz}$. The target detection probability is set as 0.9 in the opportunistic spectrum access scheme. We set $g_{1}=N_{0}=0 \mathrm{~dB}$, $q_{0}=0.7, \bar{I}=P_{p}=0.5, \bar{P}=10 \mathrm{~dB}, \gamma=h=g_{2}=0 \mathrm{~dB}$, unless otherwise mentioned.

Figure 2 compares the power allocations under the conventional strategies as well as the proposed one. The figure shows that $P_{i}$ for the proposed strategy is a non-increasing function of the received signal energy. When $x$ is small, the proposed strategy allocates more power than the conventional ones, while when $x$ is large, it allocates less power, thus the average transmit powers are the same for all the strategies.

Figure 3 shows the average secondary achievable rate. In the low $\bar{P}$ region, the proposed strategy and the conventional ones have the same rates. However, when $\bar{P}$ is high, the proposed strategy achieves much higher rates. The rates of all strategies flatten out when $\bar{P}$ is sufficiently large since the rate is decided by $\bar{I}$ under this condition. When $M$ increases, the rate of the proposed strategy becomes larger, but the gain does not improve much when $M$ is large. As $M$ becomes extremely large, say $M=1,000$ in the figure, the rate approaches an upper limit. In practice, we can choose the right $M$ to tradeoff the system complexity and performance, and in this example $M=4$ serves as a good choice.

\section{Conclusions}

In this paper, we propose a novel multiple-level power allocation strategy for $\mathrm{SU}$ in a $\mathrm{CR}$ system. The receiving signal energy from PU is divided into different categories and SU transmits with different power for each category. The power levels at SU are obtained by maximizing the average achievable rate under the constraints of the average transmit power at SU and the average interference temperature to PU. Compared with the conventional power allocation strategies, the proposed scheme offers significant rate improvement for SU. Furthermore, we are working on extending the idea to the cases of multiple SUs and statistic channel information and on how to decrease the computational complexity.

\section{Endnote}

a Namely, we have multiple thresholds to categorize $x$ rather than only using $\rho$ as did in convention.

Competing interests

The authors declare that they have no competing interests.

\section{Acknowledgements}

This work was supported in part by the National Basic Research Program of China (973 Program) under Grant 2013 CB336600 and Grant 2012CB316102, by the Beijing Natural Science Foundation under Grant 4131003, by the National Natural Science Foundation of China under Grant 61201187, by the Importation and Development of High-Caliber Talents Project of Beijing Municipal Institutions under Grant YETP0110; by the Scientific Research Foundation for the Returned Overseas Chinese Scholars, Ministry of Education, by the Tsinghua University Initiative Scientific Research Program under Grant 20121088074, and by NEC Research Fund. 


\section{Author details}

${ }^{1}$ Nanjing Institute of Electronics Engineering, Nanjing 210007, People's Republic of China. ${ }^{2}$ Department of Automation, Tsinghua University, State Key Lab of Intelligent Technologies and Systems, Tsinghua National Laboratory for Information Science and Technology (TNList), Beijing 100084, People's Republic of China. ${ }^{3}$ NEC Laboratories China, Beijing 100084, People's Republic of China.

Received: 15 February 2014 Accepted: 24 March 2014

Published: 14 April 2014

\section{References}

1. Q Wu, G Ding, J Wang, YD Yao, Spatial-temporal opportunity detection for spectrum-heterogeneous cognitive radio networks: two-dimensional sensing. IEEE Trans. Wirel. Commun. 12, 516-526 (2013)

2. S Stotas, A Nallanathan, On the throughput and spectrum sensing enhancement of opportunistic spectrum access cognitive radio networks. IEEE Trans. Wirel. Commun. 11, 97-107 (2012)

3. XW Gong, SA Vorobyov, C Tellambura, Optimal bandwidth and power allocation for sum ergodic capacity under fading channels in cognitive radio networks. IEEE Trans. Signal Process. 59, 1814-1826 (2011)

4. RF Fan, J Hai, Q Guo, Z Zhang, Joint optimal cooperative sensing and resource allocation in multichannel cognitive radio networks. IEEE Trans. Veh. Technol. 60, 722-729 (2011)

5. X Kang, YC Liang, HK Garg, L Zhang, Sensing-based spectrum sharing in cognitive radio networks. IEEE Trans. Veh. Technol. 58, 4649-4654 (2009)

6. J Ma, GD Zhao, Y Li, Soft combination and detection for cooperative spectrum sensing in cognitive radio networks. IEEE Trans. Wireless Commun. 7, 4502-4507 (2008)

7. GR Ding, QH Wu, YD Yao, JL Wang, YY Chen, Kernel-based learning for statistical signal processing in cognitive radio networkstheoretical foundations, example applications, and future directions. IEEE Signal Process. Mag. 30, 126-136 (2013)

8. GR Ding, QH Wu, F Song, JL Wang, Spectrum sensing in opportunity-heterogeneous cognitive sensor networks: how to cooperate? IEEE Sensors J. 13, 4247-4255 (2013)

9. S Stotas, A Nallanathan, Optimal sensing time and power allocation in multiband cognitive radio networks. IEEE Trans. Commun. 59, 226-235 (2011)

10. YY Pei, YC Liang, YC Teh, KH Li, How much time is needed for wideband spectrum sensing. IEEE Trans. Wirel. Commun. 8, 5466-5471 (2009)

11. RO Schmidt, A Signal Subspace Approach to Multiple Emitter Location and Spectral Estimation. Dissertation, Stanford University, 1981

12. H Liu, U Tureli, A high-efficiency carrier estimator for OFDM communications. IEEE Commun. Lett. 2, 104-106 (1998)

13. SP Lloyd, Least-square quantization in PCM. IEEE Trans. Inform. Theory. IT-28, 129-137 (1982)

14. V Lau, Y Liu, TA Chen, On the design of MIMO block-fading channels with feedback-link capacity constraint. IEEE Trans. Commun. 52, 62-70 (2004)

15. S Body, L Vandenberghe, Convex Optimization. (Cambridge University, Cambridge, 2005)

16. DP Bertsekas, Convex Analysis and Optimization. (Athena Scientific, Massachusetts, 2003)

doi:10.1186/1687-6180-2014-51

Cite this article as: Chen et al:: Multiple-level power allocation strategy for secondary users in cognitive radio networks. EURASIP Journal on Advances in Signal Processing 2014 2014:51.

\section{Submit your manuscript to a SpringerOpen ${ }^{\odot}$ journal and benefit from:}

- Convenient online submission

- Rigorous peer review

- Immediate publication on acceptance

- Open access: articles freely available online

- High visibility within the field

- Retaining the copyright to your article

Submit your next manuscript at $\boldsymbol{\nabla}$ springeropen.com 